\title{
A METHOD OF TOMATO IMAGE SEGMENTATION BASED ON MUTUAL INFORMATION AND THRESHOLD ITERATION
}

\author{
Hongxia $\mathrm{Wu}$, Mingxi $\mathrm{Li}^{*}$ \\ Huangshi Institute of Technology,Huangshi, Hubei Province, P.R.China 435003 \\ * Corresponding author, Address: Editorial Department of Journal of Huangshi Institute of \\ Technology,Huangshi 430003, Hubei Province, P. R. China, Tel: 15972372916, Email: \\ limx10920@yahoo.com.cn
}

\begin{abstract}
Threshold Segmentation is a kind of important image segmentation method and one of the important preconditioning steps of image detection and recognition, and it has very broad application during the research scopes of the computer vision. According to the internal relation between segment image and original image, a tomato image automatic optimization segmentation method (MI-OPT) which mutual information associate with optimum threshold iteration was presented. Simulation results show that this method has a better image segmentation effect on the tomato images of mature period and little background color difference or different color.
\end{abstract}

Keywords: Image segmentation, mutual information, threshold optimization, tomato image

\section{INTRODUCTION}

Traditional threshold method has only considered gray levels but neglect spatial distribution of gray scale and internal relation between segment and original of the image, such as Otsu algorithm, fuzzy $\mathrm{C}$ means algorithm(FCM), adaptive algorithm(Rigau et al.,2004;Zhou Xiaozhou et al.,2007;Lv Qingwen et al.,2006). When lighting is not uniform and noise out-burst or larger background gray scale changed, massive information will lost and the area obtained can't represent the shape of original subject after

\footnotetext{
Please use the following format when citing this chapter:

Wu, H. and Li, M., 2009, in IFIP International Federation for Information Processing, Volume 294, Computer and Computing Technologies in Agriculture II, Volume 2, eds. D. Li, Z. Chunjiang, (Boston: Springer), pp. 1097-1104.
} 
segmentation, besides FCM algorithm has the problems such as depended on initial value too much, converge on local maxima and classification number should be pre-determined, and people still can't solve these after a lot of research and improvement. So according to the internal relation between segment image and original image, a tomato image automatic optimization segmentation method which mutual information associate with optimum threshold iteration was presented.

\section{OPTIMUM THRESHOLD SEGMENTATION ALGORITHM BASED ON MUTUAL INFORMATION}

\subsection{Image mutual information measures}

Mutual information of image $\mathrm{A}$ and $\mathrm{B}$ can be defined:

$$
I(A, B)=H(A)+H(B)-H(A, B)
$$

$\mathrm{H}(\mathrm{A})$ and $\mathrm{H}(\mathrm{B})$ is the average information quantity of image $\mathrm{A}$ and $\mathrm{B}$, and $\mathrm{H}(\mathrm{A}, \mathrm{B})$ is their correlated average information quantity.

The average information quantity and correlated average information quantity of A and B can be calculated as follows:

$$
\left\{\begin{aligned}
H(A) & =\sum_{a}-p_{A}(a) \log p_{A}(a) \\
H(B) & =\sum_{b}-p_{B}(b) \log p_{B}(b) \\
H(A, B) & =\sum_{a, b}-p_{A, B}(a, b) \log p_{A, B}(a, b)
\end{aligned}\right.
$$

$P_{A}(a)$ and $P_{B}(b)$ is the probability density function with gray levels a of image $\mathrm{A}$ and gray levels $\mathrm{b}$ of image $\mathrm{B}, P_{A, B}(a, b)$ is the joint probability density function of image A and B. They can be calculated as follows:

$$
\left\{\begin{aligned}
p_{A, B}(a, b) & =\frac{h(a, b)}{\sum_{a, b} h(a, b)} \\
p_{A}(a) & =\sum_{a} p_{A, B}(a, b) \\
p_{B}(b) & =\sum_{b} p_{A, B}(a, b)
\end{aligned}\right.
$$


$h(a, b)$ is the joint-histogram of image $\mathrm{A}$ and $\mathrm{B}$, it means the number of the correlated point pairs with gray levels a of image A and gray levels $b$ of image B.

Mutual information doesn't balance consistency of different image directly dependent on gray levels but the probability of each image and the joint probability when two images combined. So it's not sensitive to gray change or one-to-one gray transformation, and can process the relationship between the positive and the negative image gray simultaneously.

\subsection{Optimum threshold iteration algorithm}

This algorithm chooses one approximate threshold as the initial value of the estimate, generates sub-images after image segmentation, and chooses new threshold according to the characteristic of sub-images, then segment again by using new threshold. After several circulation, incorrect pixels will minimized. The steps are as follows:

$$
T_{0}=\frac{Z_{\min }+Z_{\text {max }}}{2}
$$

(1) Select the initial threshold: choose the median of the image gray levels as the initial threshold.

$Z_{\min }, Z_{\max }$ means the minimum and the maximum of the image gray levels.

(2) Segment the image into two groups by using threshold $T_{k}$, and

$$
\begin{aligned}
& R_{1}=\left\{f(x, y) \mid f(x, y) \geq T_{k}\right\} \\
& R_{2}=\left\{f(x, y) \mid 0<f(x, y)<T_{k}\right\}
\end{aligned}
$$

(3) Calculate the gray means $Z_{1}$ and $Z_{2}$ of area $R_{1}$ and $R_{2}$, and

$$
\begin{aligned}
& Z_{1}=\frac{\sum_{f(i, j)<T_{k}} f(i, j) \times N(i, j)}{\sum_{f(i, j)<T_{k}} N(i, j)} \\
& Z_{2}=\frac{\sum_{f(i, j) \geq T_{k}} f(i, j) \times N(i, j)}{\sum_{f(i, j) \geq T_{k}} N(i, j)}
\end{aligned}
$$

$f(i, j)$ is the gray level of $(i, j)$ point, $N(i, j)$ is the weight coefficients of $(i, j)$, usually $N(i, j)=1.0$.

(4) Calculate new threshold $T_{k+1}$ :

$$
T_{k+1}=\frac{Z_{1}+Z_{2}}{2}
$$

(5) If $T_{k}=T_{k+1}$, then circulation ends, otherwise $k=k+1$, turns to step 2.

$T_{k+1}$ is the optimum segment threshold after circulation ends. 


\subsection{Optimization segmentation algorithm}

Mutual information Registration is the best creation of the retrospective registration method of registration accuracy and robustness, segmentation can be regarded as the image degeneration, and segment can be treated as a kind of special form, mutual information reaches maximum when spatial location of segment and original image is consistent and calculated area matches original subject's shape, and optimization segmentation results can be considered contains the most original image information quantity. So, image segmentation algorithm based on mutual information and optimal threshold iteration can be described: assume $I, I_{T}$ is original and image segmented choose initial value $T$ which is determined by using optimal threshold iteration algorithm, maximum mutual information quantity is optimal goal, searching the optimal threshold $T_{\text {optimal }}$ in the range $[T-\delta, T+\delta]:$

$$
T_{\text {optimal }}=\arg \max \left(M I\left(I, I_{T}\right)\right)
$$

Its algorithm flow shows as Fig. 1

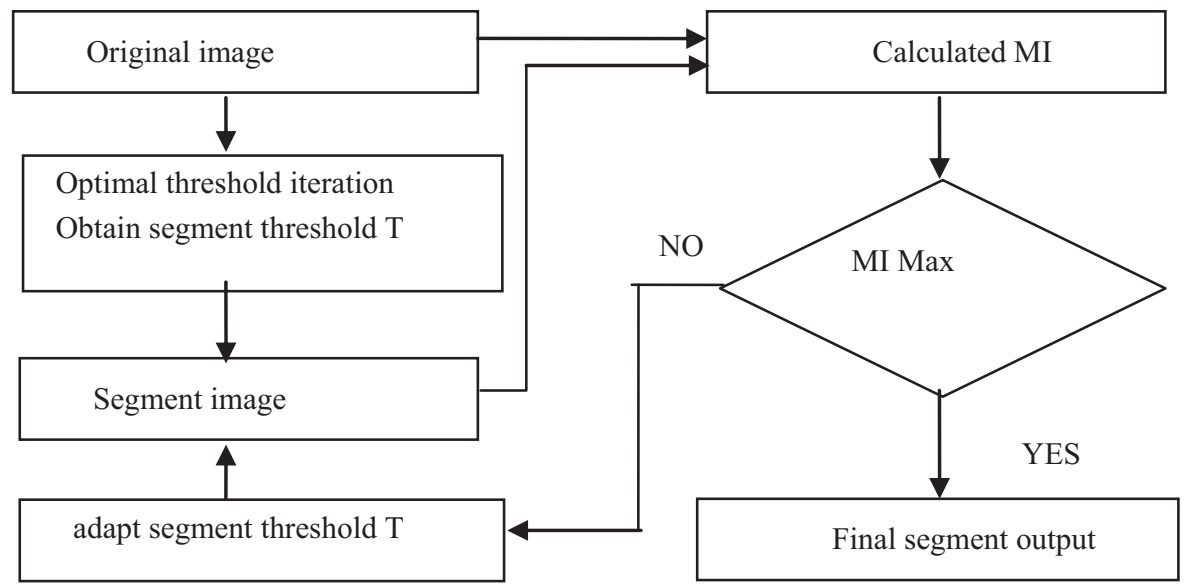

Fig. 1. the flow chart of automatic optimized segment algorithm

\section{TOMATO IMAGE SEGMENTATION COMPARISON EXPERIMENT}

According to the segmentation principle above, fig $2 \sim 6$ gives three different tomato segment results of different growing status and color by using Otsu algorithm, EN-2D algorithm(Zhang Honglei et al., 2007; Vincent, 
1993; Gao Hai et al.,2006)and MI-OPT algorithm. For better visual effect, each object contour of binary processed images stack $\mathrm{s}$ on original images in every picture (black line border of left images).
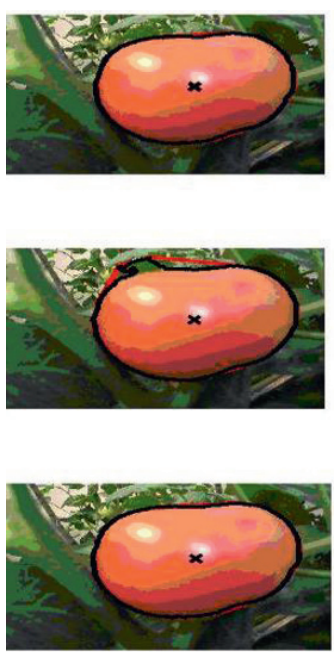

Fig. 2. simple fruit color consistent segment result
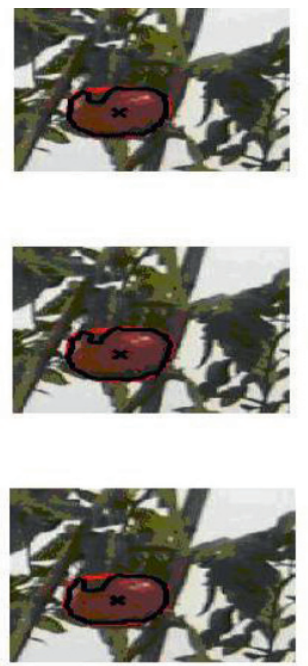

Fig. 3. slight mask segment result

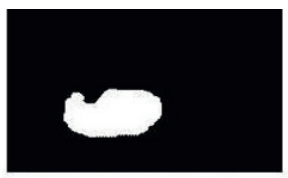

OTSU segment result

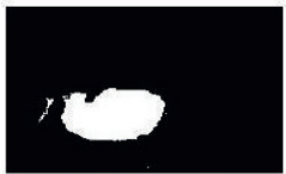

EN-2D segment result

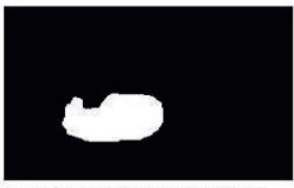

MI-OPT segment result 

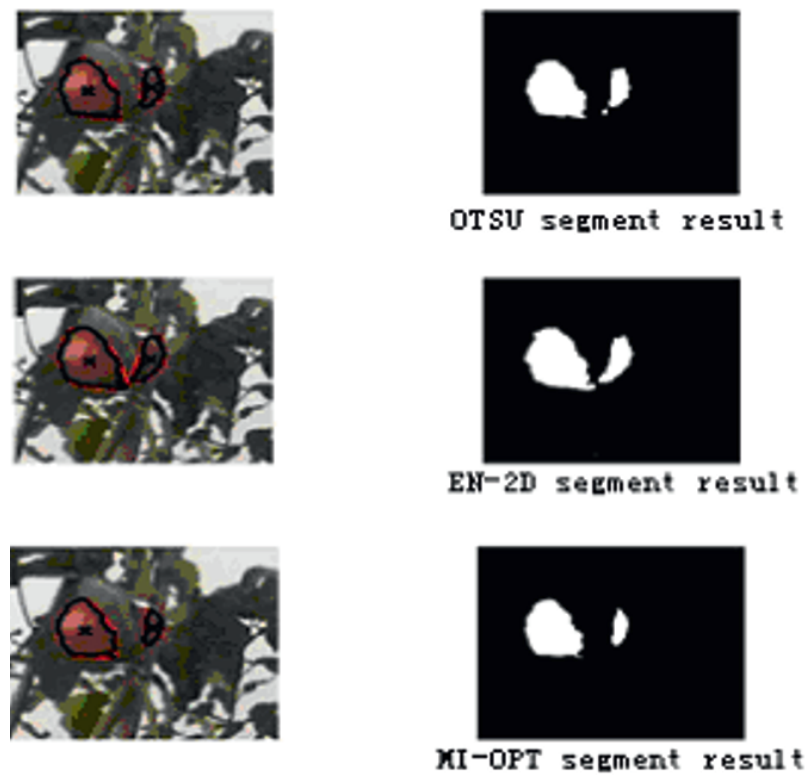

Fig.4. segment result of the serious mask fruit
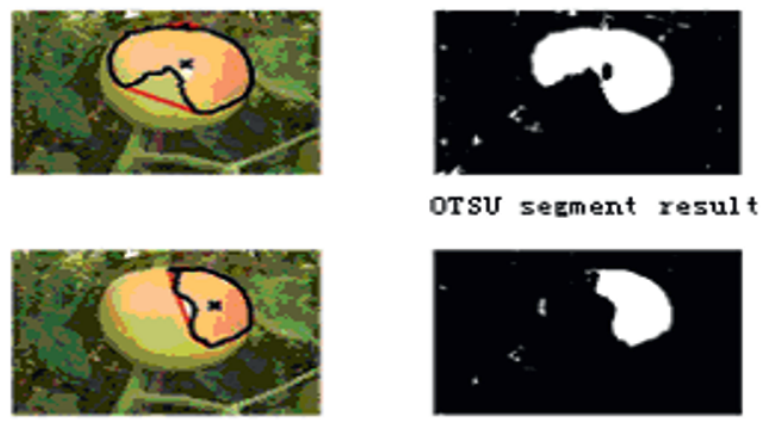

EH-2D segment result
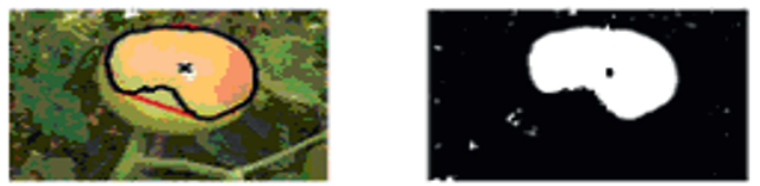

XI-OPT serment result

Fig.5. segment result of the color inconsistent fruit 

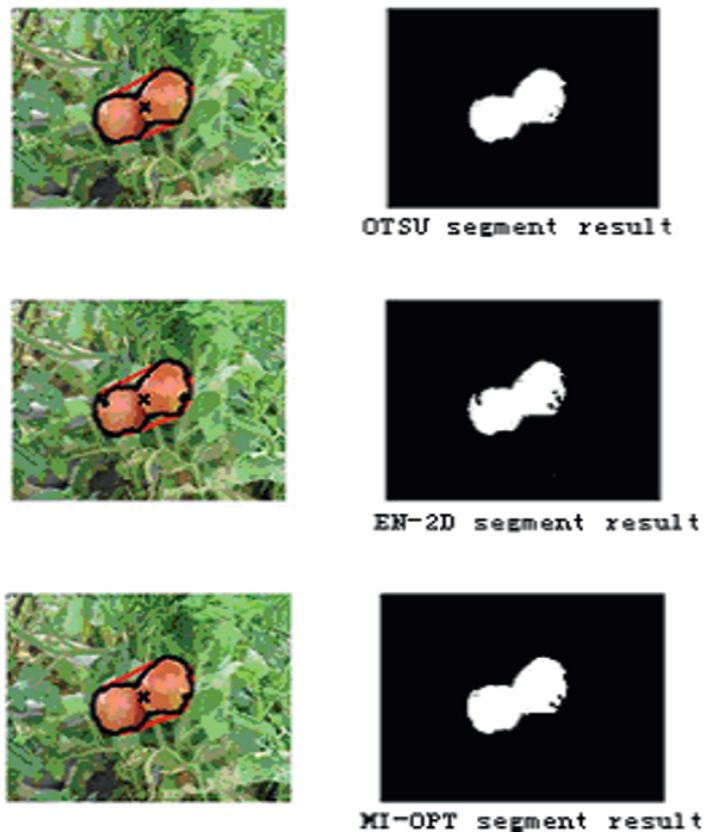

Fig.6. segment result of connected fruits
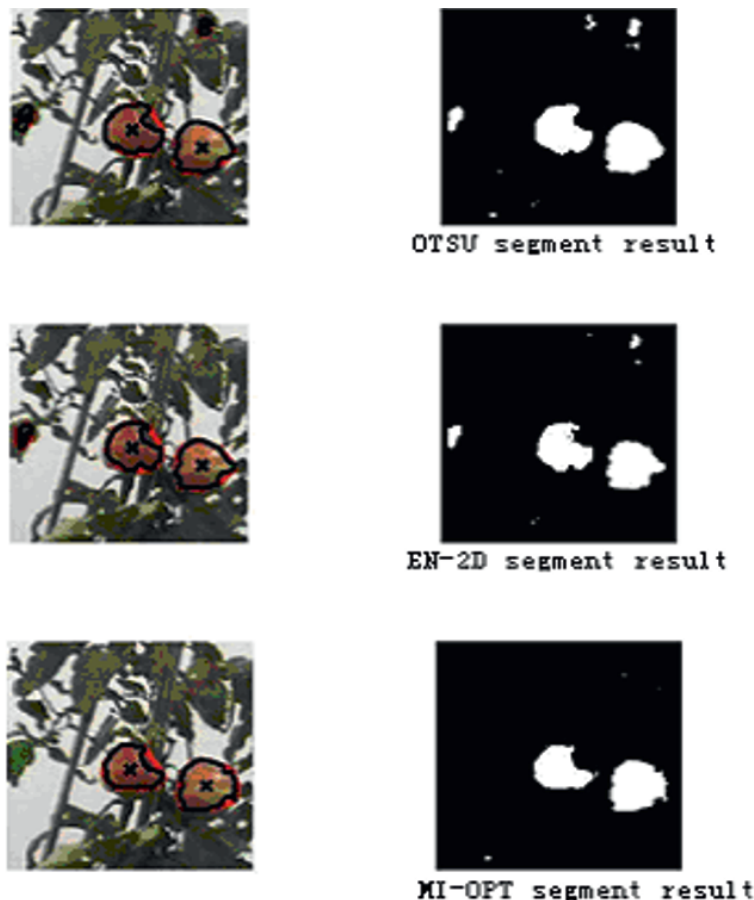

Fig. 7. segment result of color inconsistent fruits 
All these results shows that Otsu maximum between-cluster variance segmentation method based on image color difference, 2D-maximum entropy segmentation method based on image color difference and image automatic optimization segmentation method which mutual information associate with optimum threshold iteration can all segment ripe tomato images which has consistent color and separated, object contour of binary processed shows on fig 2 is relatively well-formed. To those different colors or occlusion or lapped images, all these three segmentation method can't get satisfied results, especially multi-fruit lapped and serious occlusion shows on fig 4 , and other segmentation method of these images needed to be explored.

\section{CONCLUSION}

In terms of these three segmentation method, image automatic optimization segmentation method which mutual information associate with optimum threshold iteration is better than other two, like what shows on fig3 and 5.All in all, these three self-adaptive threshold segmentation have a stable and good effect to the tomato images of larger color difference of tomato with its background and growing separately, but as to the tomato images of mature period and little background color difference or different color, image automatic optimization segmentation method which mutual information associate with optimum threshold iteration is better(shows on fig $5)$.

\section{REFERENCES}

Gao Hai, Lin Weisi, Xue Ping, et al. Marker- based image segmentation relying on disjoint set union,Signal Processing: Image Communication, 2006, 21( 2) : 100- 112

Lv Qingwen,Chen Wufan. Image Segmentation Based on Mutual Information, Chinese Journal of Computers,2006, 29 (2) : 296-301

Rigau J, Feixas M, Sbert M, et al. Medical Image Segmentation Based on Mutual Information Maximization,Proceedings of MICCAI’ 04, Saint-malo, France, 2004: 135-142

Vincent L.Morphological grayscale reconstruction in image analysis: applications and efficient algorithms,IEEE Transactions on Image Processing, 1993, 2( 2) , 176- 201

Zhang Honglei, Song Jianshe, Zhai Xiaoying.A 2D maximum-entropy based self-adaptive threshold segmentation algorithm for SAR image processing,Electronics Optics \& Control, 2007,14 (4) : 63-65

Zhou Xiaozhou,Zhang Jiawan,Sun Jizhou. Image Segmentation Method Based on Mutual Information and Chan-Vese Model ,Computer Engineering,2007, 33 (22) : 220-222 